Pacific Journal of Mathematics

A GENERALIZATION OF A THEOREM OF CHACON 


\section{A GENERALIZATION OF A THEOREM OF CHACON}

\section{Robert Chen}

A generalization of a theorem of Chacon is proved simply by an application of a maximal inequality. A pointwise convergence theorem and the submartingale convergence theorem are immediate consequences.

Let $(\Omega, \mathscr{F}, P)$ be a probability space, $\left\{X_{n}\right\}$ be a sequence of integrable random variables adapted to the increasing sequence $\left\{\mathscr{F}_{n}\right\}$ of sub $\sigma$-fields of $\mathscr{F}, B$ be the collection of all bounded stopping times (with respect to $\left\{\mathscr{F}_{n}\right\}$ ), and $D$ be the collection of random variables $Y$ which are measurable with respect to $\mathscr{F}_{x}=\sigma\left(\left\{\mathscr{F}_{n}\right\}\right)$ and, for each $w$ in $\Omega, Y(w)$ is a cluster value of the sequence $\left\{X_{n}(w)\right\}$.

The main purpose of this note is to generalize (in Theorem 1) the result stated as Corollary 1, due to Chacon ([3]). The result is a reformulation of a result due to Baxter ([2]) but our method of proof is much simpler than that in ([2]) and ([3]), and is just a simple application of a maximal inequality due to Chacon and Sucheston ([4]). A pointwise convergence theorem and the submartingale convergence theorem are immediate consequences ([1] and [5]).

THEOREM 1. Suppose that $\sup _{t \in B} E\left(\left|X_{t}\right|\right)<\infty$ and $Y_{1}, Y_{2}$ are any two random variables in $D$. Then there exist $\tau_{n}^{*}, t_{n}^{*}$ in $B$ such that $\tau_{n}^{*} \geqq n$, $t_{n}^{*} \geqq n$, and

$$
\lim _{n \rightarrow \infty} E\left\{\left|\left(X_{\tau_{n}^{*}}-X_{\tau_{n}^{*}}\right)-\left(Y_{1}-Y_{2}\right)\right|\right\}=0 .
$$

Proof. By Lemma 1 of [1] and the Borel-Cantelli lemma, for any two random variables $Y_{1}, Y_{2}$ in $D$, there exist two strictly increasing sequences $\left\{\tau_{n}\right\}$ and $\left\{t_{n}\right\}$ in $B$ such that $\lim _{n \rightarrow x} X_{\tau_{n}}=Y_{1}$ almost surely and $\lim _{n \rightarrow x} X_{t_{n}}=Y_{2}$ almost surely. By the condition that $\sup _{t \in B} E\left(\left|X_{t}\right|\right)<\infty$ and the Fatou lemma, $Y_{1}$ and $Y_{2}$ are integrable.

To prove (1), we need a maximal inequality, which I learned from Chacon and Sucheston.

(2) $\lambda P\left(\left[\sup _{n}\left|X_{n}\right| \geqq \lambda\right]\right) \leqq \sup _{t \in B} E\left(\left|X_{t}\right|\right)$ for each positive constant $\lambda$.

To see (2), let $M$ be a fixed positive integer and define a bounded 
stopping time $\tau$ by $\tau(w)=\inf \left\{n|1 \leqq n \leqq M,| X_{n}(w) \mid \geqq \lambda\right\}, \tau(w)=M+1$ if no such $n$ exists, $w \in \Omega$. Then

$$
\lambda P\left(\left[\sup _{1 \leqq n \leqq M}\left|X_{n}\right| \geqq \lambda\right]\right) \leqq E\left(\left|X_{\tau}\right|\right) \leqq \sup _{t \in B} E\left(\left|X_{t}\right|\right) .
$$

(2) follows immediately on letting $M \rightarrow \infty$.

Now, for each positive integer $k$ and each positive constant $d$, define $j(k, d)=\inf \left\{n|k \leqq n,| X_{n} \mid \geqq d\right\}, j(k, d)=\infty$ if no such $n$ exists. Let $A(k, d)=[j(k, d)<\infty]$. Since, by (2), for fixed $k, P(A(k, d)) \rightarrow 0$ as $d \rightarrow \infty, E\left\{\left|\left(Y_{1}-Y_{2}\right) \chi_{A(k, d)}\right|\right\} \rightarrow 0$ as $d \rightarrow \infty$. Therefore, for each positive integer $k$, there exists a $d_{k}$ such that $E\left\{\left|\left(Y_{1}-Y_{2}\right) \chi_{A\left(k, d_{k}\right)}\right|\right\} \leqq 1 / k$. Next, for each fixed $k$, let $Z=\max \left\{\left|X_{1}\right|,\left|X_{2}\right|, \cdots,\left|X_{k-1}\right|, \quad d_{k} \chi_{A^{c}\left(k, d_{k}\right)}+\right.$ $\left.\left|X_{f\left(k, d_{k}\right)} X_{A\left(k, d_{k}\right)}\right|\right\}, Z_{n}=X_{n \wedge ر\left(k, d_{k}\right)}$ for all $n \geqq 1$. Then it is easy to see that $\left|Z_{n}\right| \leqq Z$ for all $n \geqq 1$ and $E\{Z\}<\infty$. Since $\lim _{n \rightarrow \infty}\left(X_{\tau_{n}}-X_{t_{n}}\right)=\left(Y_{1}-Y_{2}\right)$ almost surely and, on $A\left(k, d_{k}\right), \lim _{n \rightarrow \infty}\left(Z_{\tau_{n}}-Z_{t_{n}}\right)=0$ (since $\left\{\tau_{n}\right\}$ and $\left\{t_{n}\right\}$ are strictly increasing). $\lim _{n \rightarrow \infty}\left(Z_{\tau_{n}}-Z_{t_{n}}\right)=\left(Y_{1}-Y_{2}\right) \chi_{A^{c}\left(k, d_{k}\right)}$ almost surely. Therefore, by the Lebesgue dominated convergence theorem, $E\left\{\left|\left(Z_{\tau_{n}}-Z_{t_{n}}\right)-\left(Y_{1}-Y_{2}\right) \chi_{A^{c}\left(k, d_{k}\right)}\right|\right\} \rightarrow 0$ as $n \rightarrow \infty$. Since $j\left(k, d_{k}\right) \geqq k$ and $\left\{\tau_{n}\right\},\left\{t_{n}\right\}$ are strictly increasing, we can and do choose, for each positive integer $k$, two bounded stopping times $\tau_{k}^{*}$ and $t_{k}^{*}$ in $B$ such that $\tau_{k}^{*} \geqq k$, $t_{k}^{*} \geqq k$, and $E\left\{\left|\left(X_{\tau_{k}}-X_{t_{k}}\right)-\left(Y_{1}-Y_{2}\right) \chi_{A^{c}\left(k, d_{k}\right)}\right|\right\} \leqq 1 / k$. Therefore, $\tau_{k}^{*} \geqq k$, $t_{k}^{*} \geqq k$, and $E\left\{\left|\left(X_{\tau \dot{k}}-X_{t k}\right)-\left(Y_{1}-Y_{2}\right)\right|\right\} \leqq 2 / k$ for all $k \geqq 1$. (1) follows on letting $k \rightarrow \infty$ and the proof of Theorem 1 now is complete.

COrollary 1 (Chacon). Let $\left\{X_{n}\right\}$ be a sequence of integrable random variables such that $\liminf _{n \rightarrow \infty} E\left(\left|X_{n}\right|\right)<\propto$. Then,

(3) $\quad \limsup \sup _{\tau} E\left(X_{\tau}-X_{t}\right) \geqq E\left(X^{*}-X_{*}\right)$, where $X^{*}=\limsup _{n \rightarrow x} X_{n}$, and

$$
X_{*}=\liminf _{n \rightarrow \infty} X_{n}
$$

Further, if $\sup _{t \in B} E\left(\left|X_{t}\right|\right)<\infty$, then $X^{*}$ and $X_{*}$ are integrable.

Proof. If $\sup _{t \in B} E\left(\left|X_{t}\right|\right)<\infty$, then, by Theorem $1, X^{*}, X_{*}$ are integrable and $\lim \sup _{\tau, t \in B} E\left(X_{\tau}-X_{t}\right) \geqq E\left(X^{*}-X_{*}\right)$. If $\sup _{t \in B} E\left(\left|X_{t}\right|\right)=$ $\infty$, without loss of generality, we can and do assume that $\sup _{t \in B} E\left(X_{t}^{+}\right)=$ $\infty$. Since $\liminf _{n \rightarrow \infty} E\left(\left|X_{n}\right|\right)<\infty$, there exists a strictly increasing sequence $\left\{n_{j}\right\}$ of positive integers such that $E\left(\left|X n_{j}\right|\right) \leqq M$ for all $j \geqq 1$ and some constant $M$. Now, for each bounded stopping time $t$ in $B$, let $t^{\prime}=t$ on $\left\{X_{t}^{+}>0\right\} \quad$ and $t^{\prime}=n \quad$ on $\quad\left\{X_{t}^{+}=0\right\} \quad$ where $n=\inf \left\{n_{l} \mid n_{l} \geqq\right.$ $\left.\sup \left\{t(w) \mid w \in\left\{X_{t}^{+}=0\right\}\right\}\right\}$. We then have $E\left(X_{t^{\prime}}-X_{n}\right) \geqq E\left(X_{t}^{+}\right)-M$ and 
$\sup _{\tau, t} E\left(X_{\tau}-X_{t}\right)=\infty \geqq E\left(X^{*}-X_{*}\right)$ and (3) follows immediately from this fact. The proof of Corollary 1 now is complete.

Corollary 2 (Theorem 2 of [1]). Under the conditions of Corollary 1 and consider the following two assertions:

(a) The generalized sequence $\left\{E\left(X_{t}\right) \mid t \in B\right\}$ is convergent.

(b) $X_{n}$ converges almost surely to a finite limit. Then (a) implies (b).

Corollary 3 (the submartingale convergence theorem). Suppose that $\left\{X_{n}\right\}$ is a sequence of $L_{1}$-bounded random variables adapted to the increasing sequence $\left\{\mathscr{F}_{n}\right\}$ of $\sigma$-fields. Suppose that $E\left(X_{n+1} \mid \mathscr{F}_{n}\right) \geqq X_{n}$ almost surely for all $n \geqq 1$. Then $X_{n}$ converges almost surely to a finite limit.

Remark. Corollaries 1 and 2 also hold under any one of the following two conditions.

(i) $\sup _{n} E\left(X_{n}^{+}\right)<\infty$.

(ii) $\sup _{n} E\left(X_{n}^{-}\right)<\infty$.

Acknowledgements. I would like to thank Professors Chacon and Sucheston for their valuable suggestions and comments.

\section{REFERENCES}

1. D. G. Austin, G. A. Edgar, and A. Ionescu Tulcea, Pointwise convergence in terms of expectations, Z. Wahrscheinlichkeitstheorie Verw. Gebiete, 30 (1974), 17-26.

2. J. R. Baxter, Convergence of randomly stopped variables, (to appear).

3. R. V. Chacon, A "stopped" proof of convergence, Advances in Mathematics, 14 (1974), 365-368.

4. R. V. Chacon and L. Sucheston, On convergence of vector-valued asymptotic Martingales, Z. Wahrscheinlichkeitstheorie Verw. Gebiete, 33 (1975), 55-59.

5. Ch. W. Lamb, A short proof of the Martingale convergence theorem, Proc. Amer. Math. Soc., 38 (1973), 215-217.

Received November 25, 1975.

UNIVERSITY OF MIAMI 




\section{Pacific Journal of Mathematics}

Vol. 64, No. 1

May, 1976

Walter Allegretto, Nonoscillation theory of elliptic equations of order $2 n \ldots \ldots \quad 1$

Bruce Allem Anderson, Sequencings and starters.................. 17

Friedrich-Wilhelm Bauer, A shape theory with singular homology .......... 25

John Kelly Beem, Characterizing Finsler spaces which are

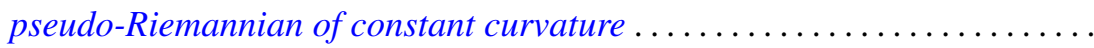

Dennis K. Burke and Ernest A. Michael, On certain point-countable

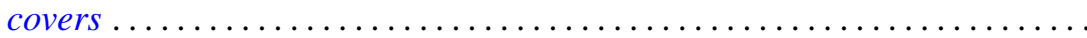

Robert Chen, A generalization of a theorem of Chacon ............... 93

Francis H. Clarke, On the inverse function theorem ................ 97

James Bryan Collier, The dual of a space with the Radon-Nikodým

property ....................................... 103

John E. Cruthirds, Infinite Galois theory for commutative rings ............ 107

Artatrana Dash, Joint essential spectra......................... 119

Robert M. DeVos, Subsequences and rearrangements of sequences in FK

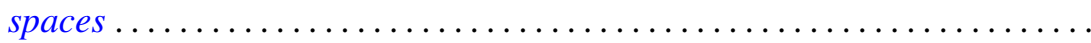

Geoffrey Fox and Pedro Morales, Non-Hausdorff multifunction generalization

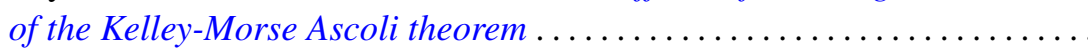

Richard Joseph Fleming, Jerome A. Goldstein and James E. Jamison, One

parameter groups of isometries on certain Banach spaces.............

Robert David Gulliver, II, Finiteness of the ramified set for branched

immersions of surfaces

Kenneth Hardy and István Juhász, Normality and the weak cb property ...... 167

C. A. Hayes, Derivation of the integrals of $L^{(q)}$-functions.

Frederic Timothy Howard, Roots of the Euler polynomials .

Robert Edward Jamison, II, Richard O'Brien and Peter Drummond Taylor, On

embedding a compact convex set into a locally convex topological vector space ....................................

Andrew Lelek, An example of a simple triod with surjective span smaller than span ...

Janet E. Mills, Certain congruences on orthodox semigroups

Donald J. Newman and A. R. Reddy, Rational approximation of $e^{-x}$ on the positive real axis.

John Robert Quine, Jr., Homotopies and intersection sequences ...

Nambury Sitarama Raju, Periodic Jacobi-Perron algorithms and fundamental units ....

Herbert Silverman, Convexity theorems for subclasses of univalent functions. . .

Charles Frederick Wells, Centralizers of transitive semigroup actions and endomorphisms of trees.........................

Volker Wrobel, Spectral approximation theorems in locally convex spaces ..................... 